\title{
THYMIC HYPERPLASIA ASSOCIATED WITH GRAVES' DISEASE: COULD THYMIC SURGERY BE DELETERIOUS?
}

\section{Monica Livia Gheorghiu', ${ }^{1}$, Elena Magheran ${ }^{3}$, Teodora Matura ${ }^{4}$, Anda Dumitrascu ${ }^{1}$}

1 "C.I. Parhon" National Institute of Endocrinology, Dept. of Endemic Goiter, Bucharest, Romania

2 "Carol Davila" University of Medicine and Pharmacy, Endocrinology, Bucharest, Romania

3 "M. Nasta" Institute for Pneumology, Dept. of Pathology, Bucharest, Romania 4 "Center for Medical Diagnosis and Treatment Roma", Bucharest, Romania

\section{INTRODUCTION}

$>$ Thymic hyperplasia is frequent (38\% of cases) in patients with Graves' disease ${ }^{1}$ (GD) but it rarely is large enough to be detected radiologically as an anterior mediastinal mass ${ }^{1,2}$. In the few operated cases, lymphoid hyperplasia (i.e. lymphoid follicle proliferation with expansion of both the cortical and the medullary thymus component) has been documented histologically in most of the cases, while true thymic hyperplasia, i.e. thymic enlargement with normal tissular architecture, was found more rarely. In only 4 out of 107 patients with GD and thymic enlargement a malignant tumor was reported (thymoma or lymphoma/leukemia) ${ }^{3}$.

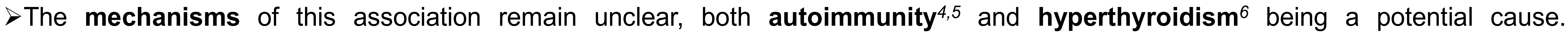
The thyrotropin receptor has been identified in the thymus ${ }^{4}$ and may be stimulated by the TSH-receptor antibodies ${ }^{5}$. Hyperthyroidism persists after thymectomy but the treatment of hyperthyroidism with antithyroid drugs usually results in a decrease of the thymus over 3-6 months ${ }^{1,7}$.

\section{OBJECTIVE}

RESULTS
To describe the evolution of 3 patients with GD and thymic enlargement detected incidentally on CT scan.

In 2 patients, a 49 years old female and a 28 years old male, the thymic mass $(3.3 / 1.6 \mathrm{~cm}$ and $5.5 / 2.5 \mathrm{~cm}$, respectively) shrinked to normal after 4 and 6 months of treatment with methymazole (MTZ). In a 37 years old female, the thymic mass $4.8 / 3.7 \mathrm{~cm}$ persisted after 5 months of MTZ and was operated.

PATIENT 2, male, 28 years old

Known with complete situs inversus, diagnosed with GD, bilateral mild exophtalmos and thymic hypertrophy occupying all the thymic lodge (CT done for persistent cough).

At diagnosis, serum TSH $=0 \mathrm{mlU} / \mathrm{L}, \mathrm{fT} 4=3.99 \mathrm{ng} / \mathrm{dL}, \mathrm{T} 3=6.65$ $\mathrm{ng} / \mathrm{mL}, \quad \mathrm{TRAB}=40 \mathrm{IU} / \mathrm{L}$ and TPOAb $>1000 \mathrm{IU} / \mathrm{mL}$. Treated with methymazole $(40 \mathrm{mg} /$ day initially $)$ and oral methylprednisolone. After 4 months of MTZ and 2 months of oral methylprednisolone in progressively lowered doses, there was a significant shrinkage of the thymus mass from 5.58/2.55/4.48 (Fig.1) to 1.3/2.9/2.9 cm (Fig 2).

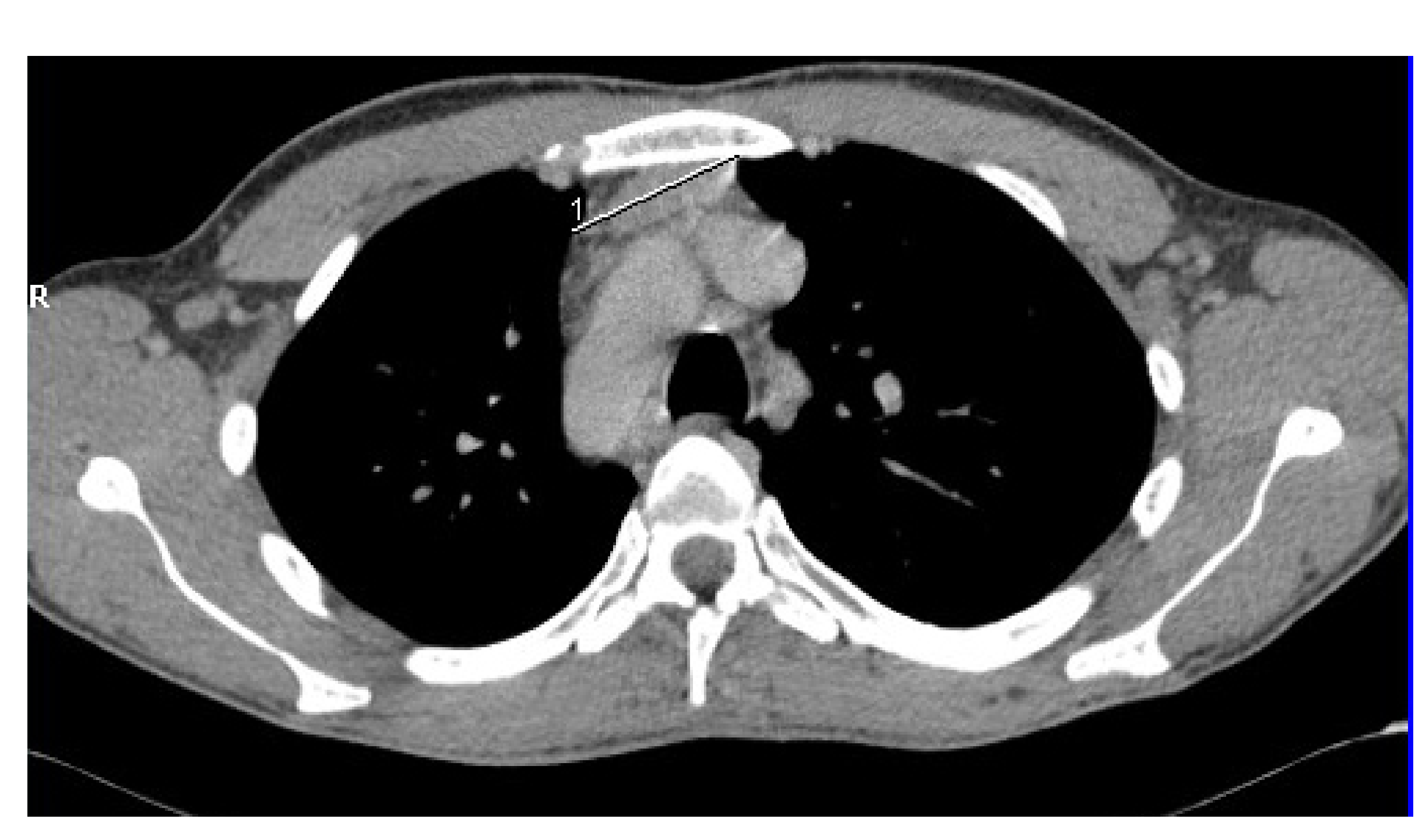

Fig. 1. CT image of well-contoured, homogenous thymus mass at the time of the diagnosis, interpreted as hyperplasia; CT was made for persistent cough.

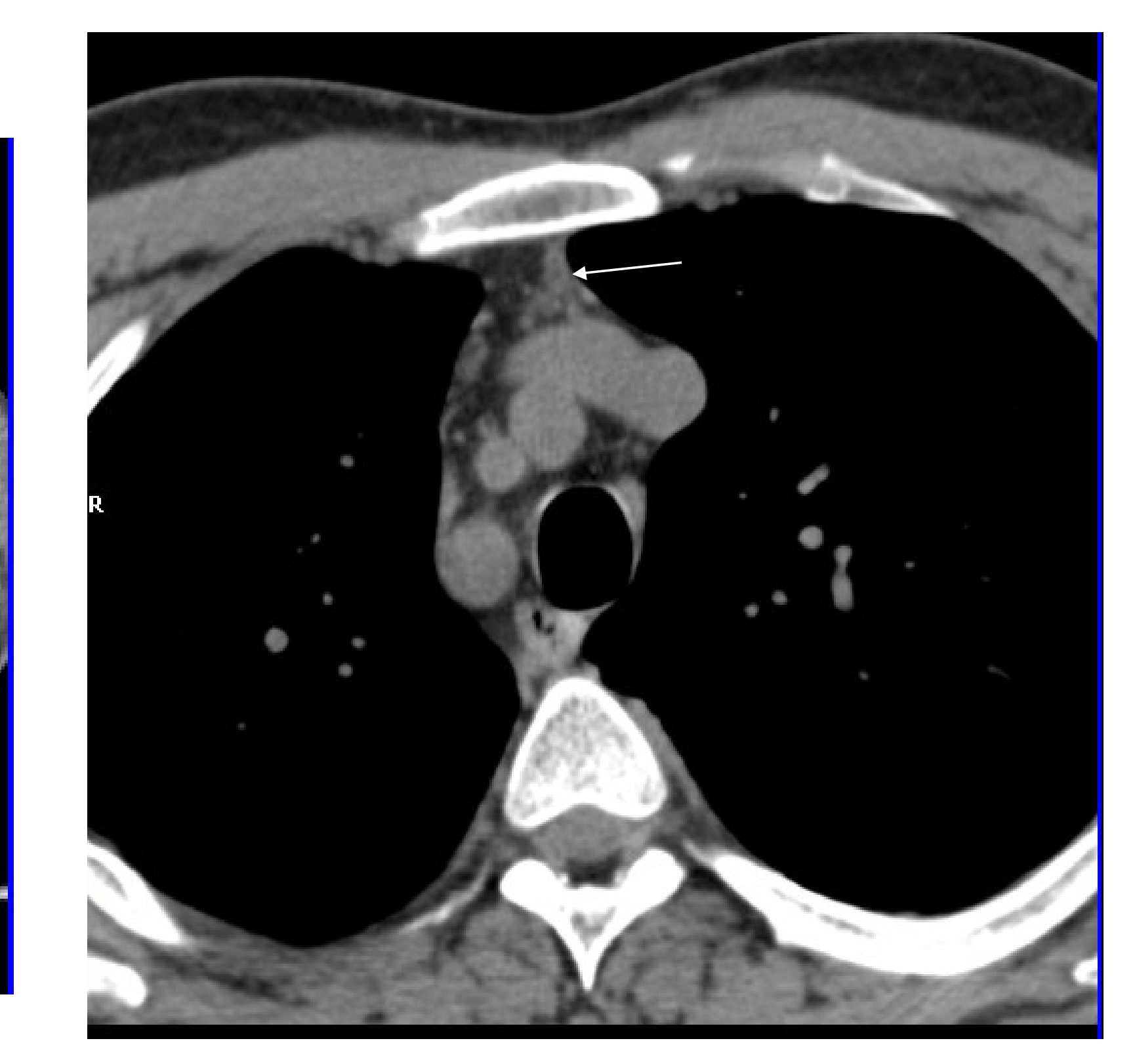

Fig. 2. CT image after 4 months of MTZ: shrinkage of the thymus mass.

PATIENT 3, female, 37 years old

GD in Apr 2014 (TSH=0,005 mmol/L, fT4=1,9 ug/dL ( $\mathrm{N}=0.9-1.71)$, TPOAb 107,8 UI/mL $(\mathrm{N}<34)$, small goiter, no exophtalmos, treated with 5-10 mg/day MTZ until Sep 2014 (when still hyperthyroid), then $15 \mathrm{mg} / \mathrm{d}$, thyroid hormones normalized in Nov 2014.

July 2014 - pneumonia, CT: homogenous thymic mass $\underline{4.8 / 3.7} \mathrm{~cm}$, lymph nodes in mediastinum, $R$ hilum and axillae (Fig 3 ).

Dec 2014 CT: homogenous stable thymic mass $\underline{3.1 / 4.1 / 3.9} \mathrm{~cm}$ (Fig 4), stable lymph nodes in the axillae.

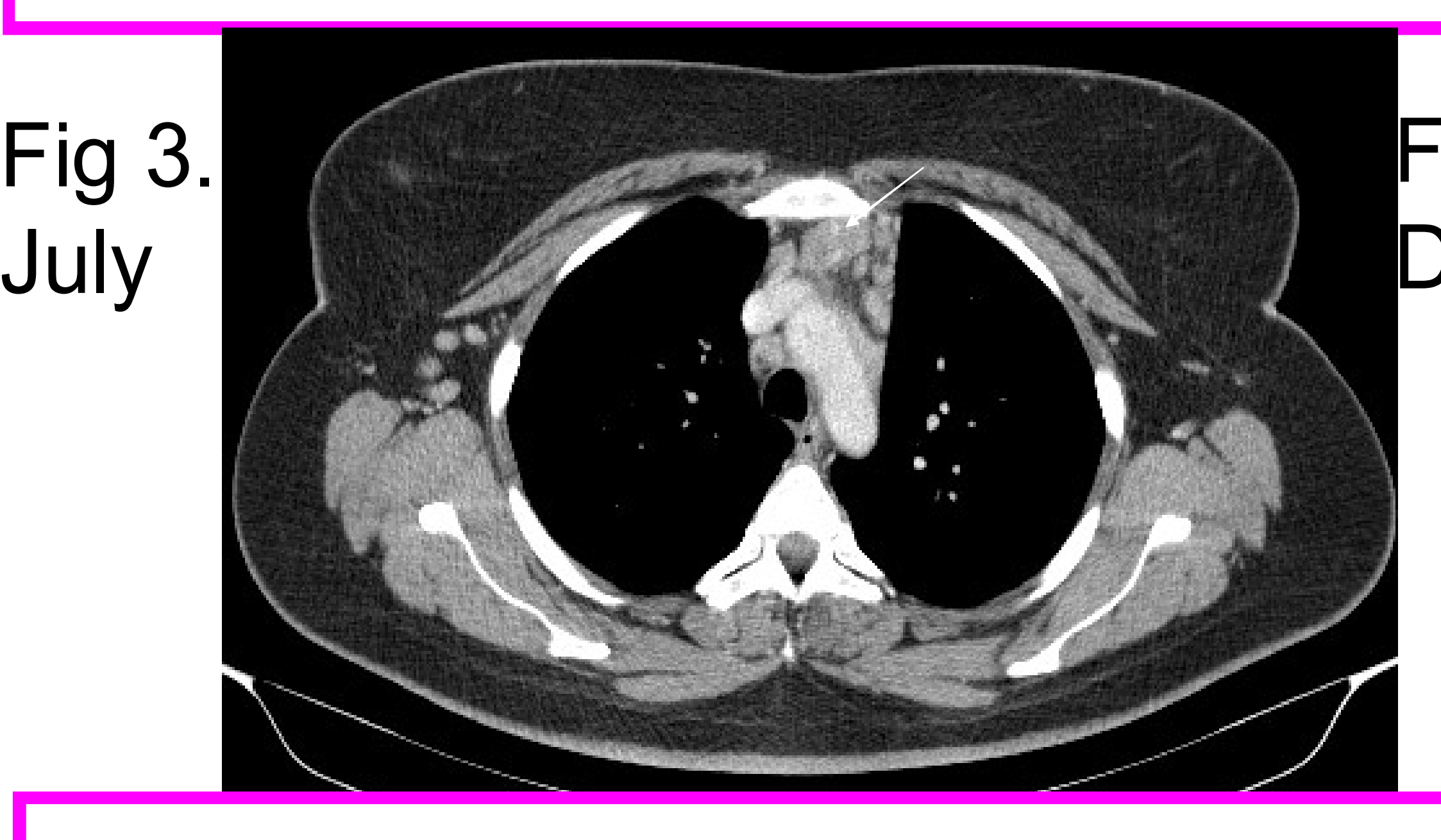

Thymic surgery,suspected thymoma HP = lymphoid hyperplasia (Fig 5)

After 2 months: miastenia gravis with frequent decompensations After 1 year: GD remission, seronegative rheumatoid arthritis After 2 years: systemic lupus eritematosus

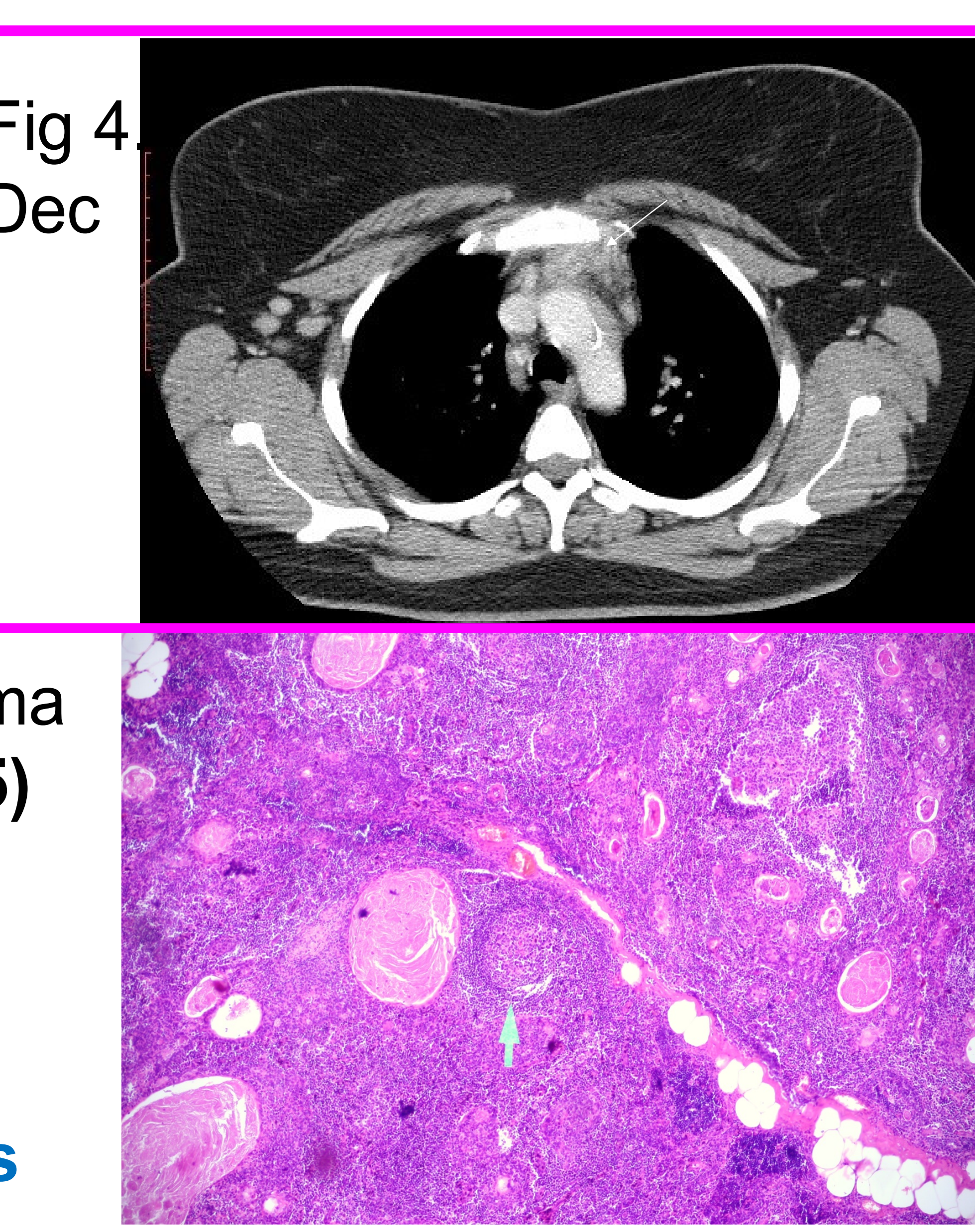

Fig 5. HE 10x, arrow: lymphoid follicle with active germinal center

\section{CONCLUSIONS}

$\checkmark$ When a thymic mass is radiologically detected in association with Graves' disease, in the absence of myasthenia gravis and/or suspect CT findings (as nonhomogeneity, invasion in the surrounding tissues, calcification, septum, cystic lesion), it usually is thymic hyperplasia.

$\checkmark$ Only antithyroid treatment and radiological follow-up are usually necessary.

$\checkmark$ By recognizing the association between thymic hyperplasia and GD and the benign course of thymic hyperthrophy after hyperthyroidism treatment, a major surgical procedure for a thymus mass, with potential risks, can be avoided.

\section{References}

${ }^{1}$ Murakami M, Hosoi Y, Negishi T, et al. J Clin Invest 1996; 98:
2228 -34
2 V. Desforges-Bullet et al. , Annales d'Endocrinologie 2011;
72: 304-309.
${ }^{3}$ Boyd JD, Juskevicius R. Thyroid Research 2012, 5:5
${ }^{4}$ Wortsman J et al. Am J Med 1988;85(1):117-21
${ }^{5}$ Song YS et al, Yonsei Med J 2016 May;57(3):795-798
${ }^{6}$ Scheiff JM, Cordier AC, Haumont S. Clin Exp Immunol
1977;27:516 -21
${ }^{7}$ Popoveniuc G, Sharma M, Devdhar M, Wexler J.A., Carroll
N.M., Wartofsky L, Burman KD. Thyroid. September 2010,
20(9):1015-1018

